\title{
Effect of the medium composition on the current of steady state voltammograms of neutral and charged species in dimethylformamide/toluene mixtures
}

\author{
M.F. Bento, M.D. Geraldo, and M.I. Montenegro* \\ Departamento de Química, Universidade do Minho, Largo do Paço, 4709, Braga Codex, Portugal. \\ *montenegro@quimica.uminho.pt
}

\begin{abstract}
Steady state voltammetric currents of both neutral and charged species obtained at microelectrodes are affected by the medium composition. Experiments carried out in N,N-dimethylformamide (DMF) and toluene/DMF mixtures for the reduction of diciano(fluoren-9-ylidene)methane (DCN) and the methylviologen cation $\left(\mathrm{MV}^{2+}\right)$ and for the oxidation of ferrocene $(\mathrm{Fc})$, in the presence of different electrolyte concentrations indicate that both viscosity of the solution and mass transport by migration may have a strong effect on the measured limiting currents. These observations are particularly important in electroanalysis since the appropriate choice of the medium may substantially improve both the sensitivity and the detection limits of the electrochemical method.
\end{abstract}

Keywords: Microelectrodes; Diffision coefficient; Viscosity; Migration

\section{Introduction}

It is well recognized that microelectrodes operate in resistive media allowing experiments to be carried out in very low dielectric constant solvents or in the absence of deliberately added electrolyte [1]. This fact has considerably widened the spectrum of electrochemical experiments and it has been possible to perform novel electroanalytical determinations under conditions considered impossible until the advent of these very small electrodes.

Changes in the solvent or the solvent composition (when mixtures of solvents are used) $[2,3]$ and in the electrolyte concentration [4], yield different limiting currents in steady state voltammograms for the same concentration of electroactive species. The observed differences may be explained in terms of changes in the medium viscosity that affect the value of the diffusion coefficient. It should be noticed that the limiting current at microdisc electrodes is given by [5],
$\mathrm{I}_{\mathrm{L}}=4 \mathrm{nFDcr}$

where $\mathrm{n}$ is the number of electrons involved per molecule/ion, D the diffusion coefficient, $\mathrm{c}$ the concentration of the electroactive species and $r$ the radius of the microdisc. The current is directly proportional to $\mathrm{D}$ (and not to $\mathrm{D}^{1 / 2}$ as in non-steady state measurements) and, hence, changes in D affect more strongly steady state currents at microelectrodes.

In electroanalytical determinations it is desirable that the measured currents are as high as possible in order to increase sensitivity and to decrease the detection limits of the method. If the electroactive species is neutral, an increase in the current may be achieved by decreasing the medium viscosity using a different solvent or solvent mixtures. If the electroactive species is charged, a decrease in viscosity will have the same effect provided the lower viscosity solvent or solvent mixture has a sufficiently large dielectric constant to promote dissociation of the electrolyte to an extent that its 
concentration in the ionic form exceeds that of the electroactive species.

The variation of supporting electrolyte concentration also affects the current and this effect depends on its concentration as well as on the charge and concentration of the electroactive species.

Under conditions where migration is not expected (presence of a large excess of electrolyte or neutral redox species), a variation of the current with the electrolyte concentration is observed and this was attributed to the variation of the diffusion coefficients. Independently of the charge of the redox species a linear relation between $\mathrm{D}$ and $\sqrt{\mathrm{c}}$ was observed $[6,7,8]$, described by,

$\mathrm{D}=\mathrm{D}^{\mathrm{o}}(1-\mathrm{A} \sqrt{\mathrm{c}})$

where $\mathrm{D}^{\mathrm{O}}$ is the value of $\mathrm{D}$ at infinite dilution and A is a constant. It has been found $[3,6]$ that in organic solvents, a decrease in the electrolyte concentration results in an increase in the diffusion coefficient.

For the case of ionic species, equation (2) was established theoretically with basis on the shift from the behaviour of ideal solutions as the concentration increases [9].

Mass transport by migration will lead to an increase or decrease in current depending on the direction of the flux of the species to the electrode being the same or the opposite to that of diffusion. Thus, for the oxidation of anions or the reduction of cations an increase in current will be observed whereas the opposite effect will take place for the oxidation of cations or the reduction of anions. These changes depend on the charge of the species, the number of electrons involved and the mechanism of the electrode process.

If mass transport by migration leads to an increase in the current when the electrolyte concentration is decreased, both migration and the variation of the diffusion coefficient contribute to the current increase. When migration occurs in the opposite direction to that of diffusion, the resulting current will increase or decrease, depending on the magnitude of both contribuitions.

The changes in current associated to migration may be theoretically predicted for single electron transfer processes [6] or EE mechanisms [10], as a function of the concentration excess, $\gamma$, ([electrolyte] / [redox species]). This effect is independent of the solvent and nature of the electrolyte and redox species, depending only on the charge of both the substrate and the ions of the electrolyte.

In the present paper the above effects are presented for the reduction of DCN and $\mathrm{MV}^{2+}$ and for the oxidation of $\mathrm{Fc}$, in $\mathrm{DMF}$ and toluene/DMF mixtures in the presence of different concentrations of electrolyte at microdisc electrodes. The change in diffusion coefficient when the electrolyte concentration is varied (in excess to that of the redox species) is analysed in order to explain this effect.

\section{Experimental}

The steady state voltammetry experiments were carried out using an E\&GG PAR 273 potentiostat / galvanostat. Data acquisition and treatment was performed by an LVM $48633 \mathrm{MHz}$ computer via a GPIB-PC 2A (National Instruments) interface using the EG\&G software M270 Vers. 4.11.

The experiments were carried out in a twoelectrode cell at $298 \mathrm{~K}$. The working electrodes were gold and platinum microdiscs of radius $6 \mathrm{~mm}$ and $14 \mathrm{~mm}$, respectively and were prepared from the cross sections of metal wires of appropriate section (Goodfellow) sealed into soft glass. The electrode radii were checked using equation (1) for the oxidation of ferrocene and the value of the diffusion coefficient published elsewhere [11]. 
Electrodes were initially polished with fine emery paper and then on a moist polishing cloth with a polishing machine (Buehler, Ecomet 3) using alumina (Buehler) with decreasing grades, from 0.3 down to $0.05 \mathrm{~mm}$. Prior to each experiment the electrodes were polished with $0.05 \mathrm{~mm}$ alumina, rinsed with water and dried. The reference electrode was a Metrohm saturated calomel electrode (reference 6.0702.100) and all potentials are quoted versus this reference electrode. All solutions were degassed with a fast stream of argon (CNO, $99.999 \%)$ prior to the experiments.

The viscosity measurements were carried out at 298 K using a Technico Ostwald viscometer (reference $\mathrm{BS} / \mathrm{U}$, size $\mathrm{C}$ ), considering $\mathrm{DMF}$ as the reference liquid.

All solutions were prepared with the highest grade chemicals available in high purity $\mathrm{N}, \mathrm{N}-$ dimethylformamide (DMF, Lab-Scan, a.r., 99.8\%) or toluene (Fluka, $99.5 \%$ ). DMF was previously dried with $\mathrm{MgSO}_{4}$ for several hours and passed through an activated neutral aluminium oxide column (Merck for chromatography column, particle size 0.063 to $0.200 \mathrm{~mm}$ ); it was then distilled under vacuum and collected in a Shlenck flask over $4 \AA$ molecular sieves. Toluene was transferred, through a transfer needle under argon, into an erlenmeyer containing $4 \AA$ molecular sieves where it was stored. Ferrocene (BDH Chemicals Ltd.) was purified by sublimation. Diciano(fluoren9-ylidene)methane (DCN) was prepared by the Knoevenagel condensation [12] from 9-fluorenone and malonitrile and recrystallized from ethylacetate / petroleum ether (40/60). N,N'-dimethyl-4,4'bipiridinium hexafluorophosphate $\left(\mathrm{MVPF}_{6}\right)$ was prepared from aqueous solutions of the corresponding chloride salt (Sigma) and $\mathrm{NH}_{4} \mathrm{PF}_{6}$ (Merck); the product was washed with distilled water and dried.

The supporting electrolyte, $\mathrm{Bu}_{4} \mathrm{NBF}_{4}$, was prepared by mixing aqueous solutions of $\mathrm{NaBF}_{4}$
(Fluka, purity $>98 \%$ ) and $\mathrm{Bu}_{4} \mathrm{NHSO}_{4}$ (Merck, purity $>98 \%$ ). The product was recrystallyzed in ethyl acetate + petroleum ether and dried under vacuum.

\section{Results and Discussion}

Figures 1 and 2 report the effect of the medium composition on the steady state voltammetric currents for the oxidation of ferrocene and the reduction of $\mathrm{MV}^{2+}$, at gold and platinum microelectrodes, respectively.

Figure 1 shows steady state voltammograms for the oxidation of ferrocene in different toluene/DMF mixtures for the same electrolyte concentration. It is clear that the limiting current increases with toluene content most probably due to a decrease in the viscosity of the solvent mixture.

The effect of varying the electrolyte concentration in the oxidation of ferrocene and the reduction of $\mathrm{MV}^{2+}$ is illustrated in figure 2. The voltammograms in figure 2.a), normalized for the ferrocene concentration, were obtained in

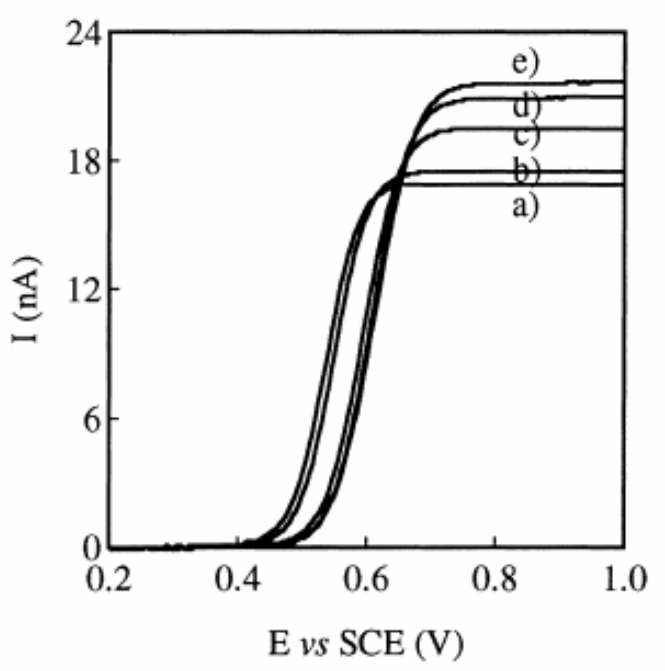

Fig. 1. Effect of the solvent composition on the steady state voltammograms for the oxidation of ferrocene, $8.01 \mathrm{mM}$, in several toluene/DMF mixtures containing $0.10 \mathrm{M} \mathrm{Bu} \mathrm{NBF}_{4}$. Percentage of toluene: (a) 0; (b) 10; (c) 50; (d) 70; (e) 90. Gold microdisc $(\mathrm{r}=6 \mu \mathrm{m})$; scan rate: $50 \mathrm{mV} / \mathrm{s} ; \mathrm{T}=298 \mathrm{~K}$. 
Table 1. Current enhacement due to the variation of solvent composition. $\Delta \mathrm{I}$ is the total variation of current. $\Delta \mathrm{I} / 0.1 \mathrm{cP}$ is the variation per $0.1 \mathrm{cP}$ of the solution viscosity.

\begin{tabular}{cccc}
\hline Redox species & $\begin{array}{c}\text { Solvent composition } \\
\text { (solution viscosity) }\end{array}$ & $\begin{array}{c}\Delta \mathrm{I} \\
(\%)\end{array}$ & $\begin{array}{c}\Delta \mathrm{I} / 0.1 \mathrm{cP} \\
(\%)\end{array}$ \\
\hline $\mathrm{Fc}$ & $\begin{array}{c}0-90 \% \text { toluene } \\
(0.871-0.749 \mathrm{cP})\end{array}$ & 29 & 23 \\
$(8.01 \mathrm{mM})$ & $\begin{array}{c}0-90 \% \text { toluene } \\
\mathrm{DCN}\end{array}$ & 34 & 28 \\
$(2.01 \mathrm{mM})$ & $(0.871-0.749 \mathrm{cP})$ & 34 & 28 \\
\hline
\end{tabular}

$90 \%$ toluene solutions containing different concentrations of $\mathrm{Bu}_{4} \mathrm{NBF}_{4}$. These voltammograms do not show the same limiting current as it would be predicted by equation (1) and the observed variations may be explained by an increase in the diffusion coefficient of ferrocene as the electrolyte concentration diminishes.

Figure 2.b) compares steady state voltammograms for the one-electron reduction of $\mathrm{MV}^{2+}$ in $\mathrm{DMF}$ containing different concentrations of $\mathrm{Bu}_{4} \mathrm{NBF}_{4}$. The observed increase in the limiting currents as the electrolyte concentration decreases, in this case, may also be explained by a migration effect. To analyse the effect of the solvent composition on the voltammetric current, viscosity measurements were

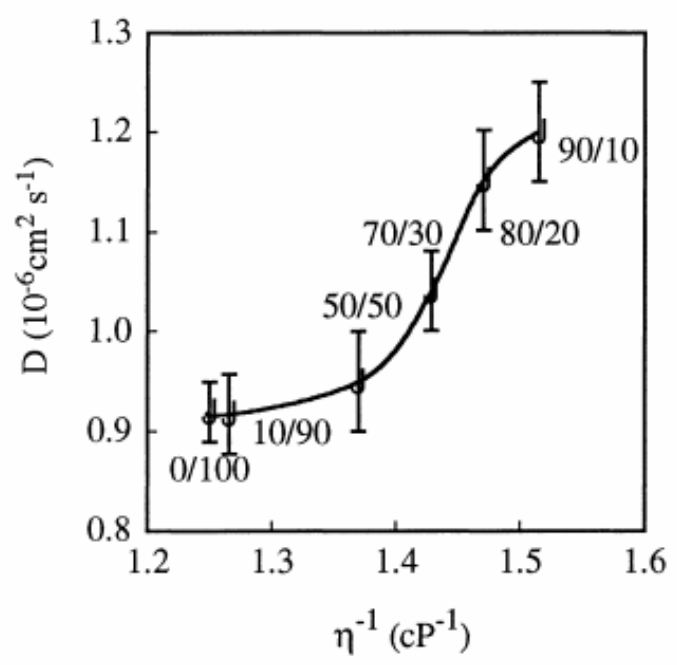

Fig. 2. Plot of diffusion coefficient of ferrocene versus the reciprocal of viscosity, according to the Stokes-Einstein equation, for a series of solvent mixtures containing $0.10 \mathrm{M}$ $\mathrm{Bu}_{4} \mathrm{NBF}_{4}$. The numbers in the plot refer to the solvent composition ( $\%$ toluene $/ \% \mathrm{DMF})$.
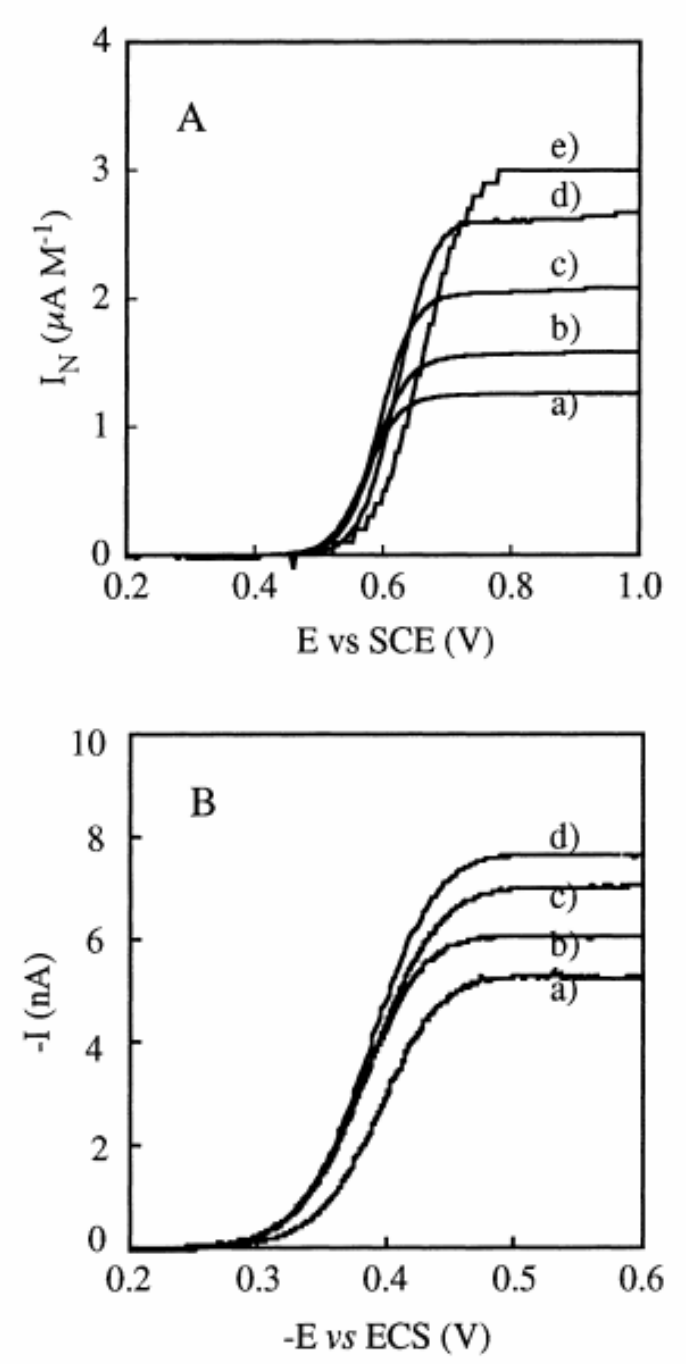

Fig. 3. Effect of the electrolyte concentration on the steady state voltammograms for: (A) the oxidation of ferrocene in different concentrations at a gold microdisc $(\mathrm{r}=6 \mu \mathrm{m})$ in $90 \%$ toluene $/ 10 \%$ DMF. $\left[\mathrm{Bu}_{4} \mathrm{NBF}_{4}\right]$ : (a) 0.80; (b) 0.60; (c) 0.40; (d) 0.20; (e) 0.050 $M, \gamma=50$. The currents are normalized for the concentration of ferrocene. Scan rate: $50 \mathrm{mV} / \mathrm{s} ; \mathrm{T}=298 \mathrm{~K}$. (B) The reduction of $\mathrm{MV}^{2+}, 2.00 \mathrm{mM}$, at a platinum microdisc $(\mathrm{r}=14 \mu \mathrm{m})$ in $\mathrm{DMF}$ containing different $\mathrm{Bu}_{4} \mathrm{NBF}_{4}$ concentrations: (a) 0.10; (b) 0.010; (c) $1.0 \times 10^{-3}$; (d) $1.0 \times 10^{-5}$ M. $0.005>\gamma>50$; scan rate: $50 \mathrm{mV} / \mathrm{s}$; $\mathrm{T}=298 \mathrm{~K}$. 
carried out. As the toluene content increases in the mixture, the solution viscosity diminishes as expected, since the viscosity of toluene $(0.560 \mathrm{cP})$ is lower than that of DMF $(0.802 \mathrm{cP})$ [13]. The plot of the medium viscosity, $\eta$, as a function of the toluene concentration is linear and follows the equation $\eta=0.873-1.38 \times 10^{-3} c_{\text {tol }}$, where $c_{\text {tol }}$ is the toluene concentration in $\%$, with a correlation coefficient of 0.998 .

The observed current variation with solvent composition depends only on the change in diffusion coefficient since no alteration is expected on the reaction mechanism, i.e., on the number of electrons involved. Hence, the current (or the diffusion coefficient) should vary with the viscosity, according to the Stokes- Einstein equation,

$\mathrm{D}=\mathrm{kT} /(6 \pi \eta a)$

where $\mathrm{k}$ is the Boltzmann constant, $\mathrm{T}$ the absolute temperature and $a$ the effective radius of the molecule. A linear relation between $D$ and $1 / \eta$ only holds if the effective radius is not altered with the solvent composition. However, the points in figure 3 do not lay in a straight line, which suggests that the variation of $a$ with the solvent composition is not negligible. This is not surprising considering the large difference between the dielectric constants of DMF and toluene and, as a consequence, the large difference between their solvation abilities. Hence, it may be concluded that the variation of $\mathrm{D}$ is due to changes in both the viscosity and the effective radius of the species.

Table 1 shows the increase of the limiting current when the solvent changes from $100 \%$ DMF to $90 \%$ toluene/10 \% DMF. The observed effect is shown in terms of the total current variation and of the one relative to a viscosity change of $0.1 \mathrm{cP}$. The results obtained for the two systems investigated are of the same order of magnitude, about $25 \%$ / $0.1 \mathrm{cP}$.

Table 2 summarizes the variation of $\mathrm{D}$ with $\sqrt{\mathrm{c}}$ for the oxidation of ferrocene and the reduction of $\mathrm{DCN}$ and $\mathrm{MV}^{2+}$. For the charged redox species the electrolyte concentration was varied maintaining

Table 2. Correlation between the diffusion coefficient of the three redox species investigated and the electrolyte concentration.

\begin{tabular}{cccccc}
\hline Redox Species & Solvent & $\begin{array}{c}{\left[\mathrm{Bu}_{4} \mathrm{NBF}_{4}\right]} \\
(\mathrm{M})\end{array}$ & $\mathrm{D}\left(10^{-6} \mathrm{~cm}^{2} \mathrm{~s}^{-1}\right) v s \sqrt{c}\left(\mathrm{M}^{1 / 2}\right)$ & $\mathrm{r}$ & $\begin{array}{c}\text { number of } \\
\text { points }\end{array}$ \\
\hline $\begin{array}{c}\mathrm{Fc} \\
(\mathrm{g}=50)\end{array}$ & $\begin{array}{c}90 \% \text { toluene } \\
\mathrm{DCN}\end{array}$ & $0.050-0.80$ & $\mathrm{y}=15.8-11.2 \mathrm{x}$ & 0.99 & 7 \\
$\begin{array}{c}(10 \% \mathrm{DMF} \\
2.01 \mathrm{mM})\end{array}$ & $\mathrm{DMF}$ & $0.050-0.60$ & $\mathrm{y}=8.18-5.42 \mathrm{x}$ & 0.994 & 5 \\
$\begin{array}{c}\mathrm{MV}^{2+} \\
(\mathrm{g}=40)\end{array}$ & $\mathrm{DMF}$ & $0.010-0.10$ & $\mathrm{y}=5.91-3.33 \mathrm{x}$ & 0.9990 & 4 \\
\hline
\end{tabular}

Table 3. Correlation between viscosity and concentration of supporting electrolyte in DMF and $90 \%$ toluene $/ 10 \% \mathrm{DMF}$.

\begin{tabular}{ccccc}
\hline Solvent & $\begin{array}{c}{[\mathrm{Bu} 4 \mathrm{NBF} 4]} \\
(\mathrm{M})\end{array}$ & $\begin{array}{c}\mathrm{h}^{-1}\left(\mathrm{cP}^{-1}\right) \text { vs } \sqrt{\mathrm{c}} \\
\left(\mathrm{M}^{1 / 2}\right)\end{array}$ & $\mathrm{r}$ & $\begin{array}{c}\text { number of } \\
\text { points }\end{array}$ \\
\hline DMF & $2.0 \times 10^{-6}-0.20$ & $\mathrm{y}=1.25-0.417 \mathrm{x}$ & 0.993 & 6 \\
DMF & $0.10-1.0$ & $\mathrm{y}=1.46-0.937 \mathrm{x}$ & 0.9990 & 4
\end{tabular}


Table 4. Correlation between the diffusion coefficient of the three redox species investigated and the viscosity of the medium.

\begin{tabular}{|c|c|c|c|c|c|}
\hline Redox Species & Solvent & $\begin{array}{c}{\left[\mathrm{Bu}_{4} \mathrm{NBF}_{4}\right]} \\
(\mathrm{M})\end{array}$ & $\mathrm{D}\left(10^{-6} \mathrm{~cm}^{2} \mathrm{~s}^{-1}\right) v s \mathrm{~h}^{-1}\left(\mathrm{cP}^{-1}\right)$ & $\mathrm{r}$ & $\begin{array}{c}\text { number of } \\
\text { points }\end{array}$ \\
\hline $\begin{array}{c}\text { DCN } \\
(2.01 \mathrm{mM})\end{array}$ & DMF & $0.02-0.30$ & $y=-3.82+8.14 x$ & 0.99 & 6 \\
\hline $\begin{array}{l}\mathrm{MV}^{2+} \\
(\mathrm{g}=40)\end{array}$ & DMF & $0.013-0.10$ & $y=-3.96+7.91 x$ & 0.995 & 4 \\
\hline $\begin{array}{c}F c \\
(\mathrm{~g}=50)\end{array}$ & $\begin{array}{c}90 \% \text { toluene / } \\
10 \% \mathrm{DMF}\end{array}$ & $0.050-0.80$ & $y=2.61+7.43 x$ & 0.998 & 11 \\
\hline
\end{tabular}

Table 5. Current enhancement for the oxidation of ferrocene and the reduction of DCN and $\mathrm{MV}^{2+}$, resulting from changes in the electrolyte concentration.

\begin{tabular}{ccccc}
\hline Redox Species & Solvent & $\begin{array}{c}{[\mathrm{Bu} 4 \mathrm{NBF} 4]} \\
(\mathrm{M})\end{array}$ & $\begin{array}{c}\Delta \mathrm{I} \\
(\%)\end{array}$ & $\begin{array}{c}\Delta \mathrm{I} / 0.1 \mathrm{M} \mathrm{Bu} 4 \mathrm{NBF} 4 \\
(\%)\end{array}$ \\
\hline $\begin{array}{c}\mathrm{Fc} \\
(\gamma=50)\end{array}$ & $90 \%$ toluene & $0.050-0.80$ & 150 & 20 \\
$\mathrm{DCN}$ & $/ 10 \% \mathrm{DMF}$ & $0.050-0.60$ & 73 & 13 \\
$\left(\begin{array}{c}2.01 \mathrm{mM}) \\
\mathrm{MV}^{2+}\end{array}\right.$ & $\mathrm{DMF}$ & $0.010-0.10$ & 20 & 22 \\
$(\gamma=40)$ & DMF & $1.0 \times 10^{-5}-0.10$ & 45 & 45 \\
$\mathrm{MV}^{2+}$ & $\mathrm{DMF}$ & & & \\
$(0.005<\gamma<50)$ & & & \\
\hline
\end{tabular}

constant the electrolyte concentration excess in order to eliminate migration effects. Under these conditions the results obtained follow equation (2) that predicts a linear relation between $\mathrm{D}$ and $\sqrt{ } \mathrm{c}$. Whilst for charged species this is the theoretically predicted behaviour, no explanation has been found yet for such dependence when the species are neutral. With basis on the Stokes-Einstein equation, the variation of the diffusion coefficient may be attributed to changes in the viscosity or/and in the effective radius. In order to verify if these changes in $\mathrm{D}$ are due to a viscosity effect, viscosity measurements were carried out in solutions containing different electrolyte concentrations. The results obtained indicate that in both $100 \%$ DMF and $90 \%$ toluene/10\% DMF solutions, a decrease in electrolyte concentration yield a decrease in the viscosity.

Different correlations between $\eta$ and c have been tested, such as, $\left(\eta / \eta_{0^{-1}}\right) / \sqrt{ } \mathrm{c}$ vs $\sqrt{ } \mathrm{c}$ [14] and $\mathrm{c} /$ $\log \left(\eta / \eta_{\mathrm{o}}\right)$ vs c [15]. The best fitting was given by $\eta^{-1}$ vs $\sqrt{ }$ c, a linear relationship was observed in limited concentration ranges as shown in table 3 . With basis on this empirical correlation and in equation (3) it is possible to explain the observed dependence between D (or I) and the square root of the electrolyte concentration. As D is proportional to $\eta^{-1}$ and the solution viscosity varies with the concentration of the electrolyte, exhibiting a linear dependence when plotted as $\eta^{-1} v s$ the square root of the electrolyte concentration, the variation of $\mathrm{D}$ 
with $V_{c}$ will be a consequence of the viscosity change.

A good linear relationship between $\mathrm{D}$ and $\eta^{-1}$ for the DMF solutions (table 4) containing different electrolyte concentrations suggests that the effective radius of the redox species remains constant in all the solutions investigated, which is not surprising since the solvation ability is likely to be the same. The same conclusion can be withdrawn for the $90 \%$ toluene/10 \% DMF mixture.

Table 5 reports the total current variation, $\Delta \mathrm{I}$, and its variation for a $0.1 \mathrm{M}$ change in the supporting electrolyte concentration, $\Delta \mathrm{I} / 0.1 \mathrm{M}$, for the three redox species investigated, in the absence and in the presence of migration. For the oxidation of $\mathrm{Fc}$ and the reduction of $\mathrm{DCN}$, migration is always absent since these are neutral species and for the reduction of $\mathrm{MV}^{2+}$ when $\gamma>40$, the electrolyte is in a large excess so that migration is prevented. In these situations the value of $\Delta \mathrm{I} / 0.1 \mathrm{M}$ ranges between 15-20\%. However, when the electrolyte concentration decreases to values comparable to that of the redox species, the current for the reduction of $\mathrm{MV}^{2+}$ is affected by migration; when $\gamma$ is varied from 50 to 0.005 , an increase of 45 $\%$ in $\Delta \mathrm{I}$ is obtained (table 5). The current enhancement in this case is the result of both an increase in D and a migration effect. It is, then, possible to subtract to this value of $\Delta \mathrm{I}$ an amount of $22 \%$ relative to the change due to the increase in $\mathrm{D}$, and the remaining value of $23 \%$ should be only due to the migration contribution. This value is in good agreement with the theoretical prediction of $27 \%$ [6] and this fact demonstrates that the two effects can be easily deconvoluted.
The possibility of carrying out electrochemical studies in low dielectric constant solvents or in the presence of only traces of electrolyte by using microelectrodes may be explored with advantage in analytical applications. It is already recognized that working with low electrolyte concentration decreases the level of impurities in the solution, in addition to widen the available potential window.

The results presented here demonstrate a further advantage associated with the decrease of electrolyte concentration. Indeed, this leads to an increase of the steady state currents and, hence, improves the sensitivity and the detection. The increase in current with the variation of the solvent composition was attributed both to the change in the medium viscosity and to the change in the species solvation. The viscosity measurements in solutions of different electrolyte concentrations have shown that the increase in current with the decrease in the electrolyte concentration is due to the decrease in the solutions viscosity. In the case of the charged species in conditions where migration is present, it was shown that, upon correction for the current enhancement due to viscosity, the curent variation is the same as that predicted due to the migration effect. Therefore, it is possible to envisage this type of current analysis being useful in speciation studies, since the current increase or decrease due to migration is a function of the charge of the redox species.

\section{Acknowledgement}

This work has been supported by PRAXIS Programme / FEDER (Project PRAXIS/2/2.1/QUI /260/94.

\section{References}

\section{Conclusions}


[1] M.I. Montenegro, M.A. Queirós, and J.L. Daschbach, Eds., Microelectrodes: Theory and Applications, NATO ASI Series E, Vol. 197, Kluwer, Dordretch, 1991.

[2] M.J. Peña, M. Fleischmann, and N. Garrad, J. Electroanal. Chem., 220 (1987) 31.

[3] M.D. Geraldo, M.I. Montenegro, and D. Pletcher, J. Electroanal. Chem., 418 (1996) 41.

[4] M.F. Bento, M.J. Medeiros, M.I. Montenegro, C. Beriot, and D. Pletcher, J. Electroanal. Chem., 345 (1993) 273.

[5] K.B. Oldham, J. Electroanal. Chem., 122 (1981) 19.

[6] C. Amatore, B. Fosset, J. Bartlet, M.R. Deakin, and R.M. Wightman, J. Electroanal. Chem., 256 (1988) 1.

[7] S. Daniele, M. A. Baldo, M. Corbetta, and G. A. Mazzocchin, J. Electroanal. Chem., 379 (1994) 261.

[8] M. Ciszkowska, Z. Stojek, S.E. Morris, and J.G. Osteryoung, Anal. Chem., 64 (1992) 2372.
[9] J.O.'M. Bockris and A.K.N. Reddy, Modern Electrochemistry, Plenum press, New York, 1970, cap. 4.

[10] C. Amatore, M.F. Bento, and M.I. Montenegro, Anal. Chem., 67 (1995) 2800.

[11] J.E. Baur and R.M. Wightman, J. Electroanal. Chem., 305 (1991) 73.

[12] S.M. McElvain, and D.H. Clemens, in Organic Syntheses, N. Rabjohn (Ed.), Wiley \& Sons, New York, 1963, vol.4, p. 463.

[13] Handbook of Chemistry and Physics, Ed. D.R. Lide, 74 th Edition, CRC Press, London, 1993 - 1994.

[14] G. Jones and M. Dole, J. Am. Chem. Soc., 51 (1929) 2950.

[15] D. Eagland and G. Pilling, J. Phys. Chem., 76 (1972) 1902. 